\title{
2 Structural genomics on membrane proteins: comparison of more 3 than 100 GPCRs in 3 expression systems
}

4 Kenneth Lundstrom - Renaud Wagner - Christoph Reinhart •

5 Aline Desmyter - Nadia Cherouati - Thierry Magnin - Gabrielle Zeder-Lutz •

6 Melanie Courtot · Cécile Prual - Nicolas André - Gherici Hassaine ·

7 Hartmut Michel $\cdot$ Christian Cambillau $\cdot$ Franc Pattus

\begin{abstract}
Production of recombinant receptors has been one of the major bottlenecks in structural biology on $G$ protein-coupled receptors (GPCRs). The MePNet (Membrane Protein Network) was established to overexpress a large number of GPCRs in three major expression systems, based on Escherichia coli, Pichia pastoris and Semliki Forest virus (SFV) vectors. Evaluation by immunodetection demonstrated that $50 \%$ of a total of 103 GPCRs were expressed in bacterial inclusion bodies, $94 \%$ in yeast cell membranes and $95 \%$ in SFV-infected mammalian cells. The expression levels varied from low to high and the various GPCR families and subtypes were analyzed for their expressability in each expression system. More than $60 \%$ of the GPCRs were expressed at milligram
\end{abstract}

K. Lundstrom · G. Hassaine

Bioxtal, Chemin des Croisettes 22, Epalinges CH-1066, Switzerland

K. Lundstrom

e-mail: lundstrom@flamel.com

R. Wagner · N. Cherouati - T. Magnin - G. Zeder-Lutz • M. Courtot · F. Pattus

UMR7175 Department of Receptors and Membrane

Proteins, Illkirch, France

C. Reinhart · C. Prual · N. André · H. Michel

Max Planck Institute of Biophysics, Frankfurt, Germany

A. Desmyter C. Cambillau

University of Marseille, Marseille, France

Present address

K. Lundstrom ( $\square)$

Flamel Technologies, 33, Avenue du Dr. Georges Levy,

Venissieux 69693, France

e-mail: lundstrom@flamel.com levels or higher in one or several systems, compatible to structural biology applications. Functional activity was determined by binding assays in yeast and mammalian cells and the correlation between immunodetection and binding activity was analyzed.

Keywords Expression vectors $\cdot G$ protein-coupled receptors - Structural genomics

\section{Introduction}

G protein-coupled receptors (GPCRs) represent more than $50 \%$ of current drug targets, which generate more than $\$ 50$ billion in annual sales. The functions of GPCRs are many and include various signal transduction pathways but also intercellular mechanisms (Lundstrom and Chiu 2005). GPCRs can be activated through peptides, neurotransmitters, hormones, odors, ions, light, odorants, pheromones, amino acids, amines, nucleotides, nucleosides, prostaglandins and other low weight molecules. Due to the broad spectrum of functions, GPCRs play important roles in various indications such as cardiovascular, metabolic, neurodegenerative, neurological, psychiatric, viral diseases and cancers.

The success in obtaining high resolution structures of membrane proteins has in general been very modest. In comparison to the current number of more than 30,000 entries for soluble proteins in public databases, only 104 unique structures have been solved for membrane proteins (http://blanco.biomol.uci.edu/ Membrane_Proteins_xtal.html). The situation is even worse for GPCRs for which only a single structure has

\begin{tabular}{|l|ll|}
\hline Journal : 10969 & Dispatch : 11-9-2006 & Pages : 14 \\
Article No. : 9011 & $\square$ LE & $\square$ TYPESET \\
MS Code : JSFG5 & $\checkmark$ CP & $\checkmark$ DISK \\
\hline
\end{tabular}


so far been published (Palczewski et al. 2000). Even so, to obtain the structure for the bovine rhodopsin was only possible when the receptor was isolated from cow retina in an inactive conformation. However, the high receptor density of rhodopsin is unique as GPCRs only occur at low abundance. The main bottlenecks for not being successful in obtaining high resolution structures for recombinantly expressed GPCRs are the following: First, the expression of quantitatively and qualitatively acceptable recombinant receptors has been difficult. Furthermore, the expressed GPCRs need to be subjected to solubilization procedures in the presence of detergents prior to purification. This step can substantially reduce the yields of active receptor. Moreover, the GPCRs are generally extremely sensitive to degradation during the purification step, which means that the stability of purified receptor material is poor and the shelf-life short. Finally, crystallization in presence of detergents is more demanding than for soluble proteins. GPCRs also present flexible regions thereby reducing the contact sites required for appropriate crystal formation.

Although the expression levels have been fairly moderate for many GPCRs tested, in some cases relatively high receptor levels have been obtained. For instance, the human adenosine A2a receptor was expressed as a fusion protein with the maltose binding protein (MBP) in E. coli membranes with a $B_{\max }$ value of 17-34 pmol per milligram (Weiss and Grisshammer 2002). Expression in bacterial inclusion bodies has generated large quantities of GPCRs, although the necessity of complicated refolding processes has hampered the success (Lopez de Maturana et al. 2003; Baneres et al. 2003). High yields and high density of functional GPCRs (25-40 pmol/mg) has been obtained in yeast expression systems (Reinhart and Krettler 2006), particularly in Pichia pastoris (Weiss et al. 1995; André et al. 2006). Expression in insect cells from baculovirus vectors has also been commonly applied for GPCRs resulting in milligram yields of receptors per liter culture (Mazina et al. 1994). Mammalian expression has been hampered by low yields, timeconsuming production and high costs. Generally, studies have focused on a limited number of GPCRs at a time with the exception of a parallel study of 16 GPCRs in insect cells applying baculovirus vectors (Akermoun et al. 2005). For this reason, there has usually been neither any comparison between the expressability of individual receptors nor comparable studies between expression systems. The approach initiated by MePNet (Membrane Protein Network) to study 103 GPCRs overall in parallel in three wellestablished expression systems has therefore provided a unique opportunity to evaluate the expression properties of a large number of GPCRs. The selection of GPCRs was preliminary based on ligand availability to be able to monitor the functional activity of receptors expressed in yeast and mammalian cell membranes by radioligand assays and also later on to perform binding assays for purified and solubilized GPCRs as well as refolded receptors from E. coli inclusion bodies. Other criteria were to have representation of different classes and subtypes of GPCRs and to link the targets to human disease. In this context, the majority of GPCRs studied were from class A (95 GPCRs) receptors, which are represented by light (rhodopsin), adrenaline (adrenergic) and olfactory receptors. Only two members of the class B hormone and neuropetide receptors were included in the study. The class C GPCRs with the characteristically large extracellular N-terminal were represented by 4 members. Finally, two yeast receptors were from class D. Of the total number of 104 GPCRs in this study, 82 were of human origin, 9 from rat, 7 from mouse, 2 from bovine, 2 from yeast, 1 from pig and 1 from hamster.

The choice of expression systems was based on previous experience of convenience in handling a large number of clones in parallel, the potential of scale-up and obviously the possibility to obtain yields compatible to structural biology applications. E. coli-based expression was chosen because of the speed, the inexpensive process and the ease of use. The straight forward scale-up to fermentor cultures was also considered as an advantage. E. coli vectors chosen in this study were pET15 vectors with $\mathrm{N}$ - and C-terminally engineered 10-histidine tags, respectively. Fiftyfive of the 103 GPCRs were also introduced into 6 Gateway vectors containing various fusion partners. Under the expression conditions used in this study, GPCRs were always produced as insoluble inclusion bodies in the cytoplasm of $E$. coli. It was decided to use E. coli vectors in this study that uniquely promote expression in bacterial inclusion bodies although relatively high expression have been reported for GPCR expression in bacterial membranes (Tucker and Grisshammer 1996, Weiss and Grisshammer 2002). However, recent improvement in refolding of GPCRs from inclusion bodies (Baneres et al. 2003) was encouraging and the extensive need for construct engineering (mutations, deletions, evaluation of different fusion partners and tags) required for membrane-based expression was not feasible for 100 GPCRs in parallel. For this reason, pET15 vectors with $\mathrm{N}$ - and C-terminally engineered 10-histidine tags, respectively, were employed. A large portion of the 103 GPCRs were also introduced into Gateway vectors 
containing various fusion partners. Pichia pastoris vectors were applied for expression in yeast cells as a proven lower eukaryotic system. The $P$. pastoris system is well establish and fairly rapid to work with and generates large biomasses in fermentor cultures. Moreover, several GPCRs have been previously successfully expressed with this system (reviewed in Reinhart and Krettler 2006). Finally, the third system represents expression in mammalian cells. Semliki Forest virus (SFV) has previously been shown to be applicable to the expression of a large number of recombinant proteins, including many GPCRs (Lundstrom 2003). The expression levels have been very high with $B_{\max }$ values of $150-200 \mathrm{pmol} / \mathrm{mg}$ and large-scale bioreactor production in mammalian suspension cultures has been established for SFV (Blasey et al. 1997). The inclusion of members of all GPCR families and several subtypes provided additional interesting information in relation to expression patterns. The study of 103 GPCRs also allowed to describe which receptors were not successfully expressed and in which system. Finally, the expression evaluation study has provided a large number of well expressed targets that currently are subjected to further structural biology exploration. We have previously described the overexpression of 20 GPCRs from P. pastoris André et al. 2006) and 101 GPCRs from SFV-infected mammalian cells (Hassaine et al. 2006), but this is the first time a comparison of more than 100 GPCRs is presented for three expression systems.

\section{Materials and methods}

Cloning and subcloning of GPCR genes into expression vectors

Appropriate primers were designed for the $5^{\prime}$ and $3^{\prime}$ end coding regions of each GPCR, which were amplified from plasmid DNA when available or from commercial polyA ${ }^{+}$RNA preparations (Clontech, Palo Alto, CA, USA). In the latter case, RT-PCR reactions were performed to amplify the full-length ORFs using the Titan One-Step RT-PCR kit from Roche (Penzberg, Germany) according to the manufacturer's instructions. In both cases, the amplified fragments were subcloned into the pCR $4{ }^{\circledR}$ Blunt$\mathrm{TOPO}^{\circledR}$ vector (Invitrogen, Carlsbad, CA, USA) and the accuracy of the sequence verified. In case of differences from the published sequence, the existence of potential polymorphism was investigated. Mutations introduced by the RT-PCR procedure were corrected accordingly. Orientation-defined GPCR fragments with 5' end BamHI and $3^{\prime}$ end SpeI sites were obtained using type IIS restriction endonucleases (Esp31 or BbiI) or BamHI-SpeI restriction enzymes when feasible. These fragments were then subcloned into the BamHI-SpeI digested pET15N2, pET15C2, pPIC19KHisFLAGBio, pSFV2genB and pSFV2genC expression vectors (Fig. 1). For Gateway ${ }^{\mathrm{TM}}$ (Invitrogen, Carlsbad, CA, USA) cloning PCR fragments were amplified containing the respective attB1 and attB2 recombination sites into Gateway ${ }^{\mathrm{TM}}$ destination vectors. Six Gateway ${ }^{\mathrm{TM}}$ expression vectors were used (Fig. 1).

E. coli based expression in bacterial inclusion bodies

The bacterial expression vectors were transformed into four E. coli strains: BL21(DE3)pLysS and Rosetta(DE3)pLysS from Novagen and C41(DE3) and C43(DE3) from Avidis SA (Saint-Beauzire, France) (Miroux and Walker 1996). Freshly transformed bacteria were cultured in LB medium supplemented with $100 \mu \mathrm{g} / \mathrm{mL}$ ampicillin $(34 \mu \mathrm{g} / \mathrm{ml}$ clhloramphenicol for BL21(DE3)pLysS and Rosetta(DE3)pLysS) at $37^{\circ} \mathrm{C}$ until an $\mathrm{OD}_{600}$ value of $0.7-0.8$ was reached. The expression of recombinant GPCRs was induced by addition of $1 \mathrm{mM}$ IPTG (isopropyl- $\beta$-D-thiogalactopyranoside) at $37^{\circ} \mathrm{C}$ for $3 \mathrm{~h}$ or at $25^{\circ} \mathrm{C}$ or $15^{\circ} \mathrm{C}$ for $16 \mathrm{~h}$. Cells were harvested at $+4^{\circ} \mathrm{C}$ by centrifugation at $5000 \times g$ for $20 \mathrm{~min}$, lysed in $50 \mathrm{mM}$ Tris $-\mathrm{HCl}$ $\mathrm{pH} 8.0,300 \mathrm{mM} \mathrm{NaCl}, 0.1 \%$ Triton $\mathrm{X}-100,1 \mathrm{mM}$ EDTA, $0.25 \mathrm{mg} / \mathrm{ml}$ lysozyme and $1 \mathrm{mM}$ phenylmethylsulfonyl fluoride and stored at $-20^{\circ} \mathrm{C}$ prior to Western blotting. The optimal growth conditions were applied to $1 \mathrm{~L}$ flask and $8 \mathrm{~L}$ fermentor cultures. Additionally, a number of GPCRs were analyzed for the localization recombinant receptors in E. coli. Total lysates of bacteria were obtained by lysosome treatment and sonication. Inclusion bodies (pellet) were separated from soluble protein (supernatant) by centrifugation at $10,000 \times g$ for $30 \mathrm{~min}$ followed by SDS-PAGE and Western blotting.

Yeast-based expression from Pichia pastoris vectors

The yeast expression vector constructs for the 100 GPCRs were linearized with PmeI or SacI and the $P$. pastoris strain SMD1163 electroporated at $1500 \mathrm{~V}$, $25 \mu \mathrm{F}$ and $600 \Omega$ using a Gene Pulser I (Bio-Rad, Reinach, Switzerland). Selection of clones was done as previously described (Weiss et al. 1998, André et al. 2006). In conclusion, recombinant $\mathrm{His}^{+}$clones were first selected on MD agar plates $(1.34 \%$ yeast nitrogen 
Fig. 1 Schematic

presentation of expression

vectors. $\alpha \mathrm{F}$, yeast $\alpha$ Factor;

Bio, biotinylation domain

from Proprionibacterium

shermanii; FLAG, FLAG-tag;

His, 10-histidine tag; GST,

Glutathion-S-transferase; K,

Kozak consensus sequence;

MBP, maltose binding

protein; NusA, transcription

factor NusA; ss, signal

sequence from influenza HA

(hemagglutin) gene; Tev

(Tobacco Etch Virus), Tev protease cleavage site; Trx,

Thioredoxin; white triangle,

T7 promoter; grey triangle,

yeast AOX1 promoter; black

triangle, SFV 26S subgenomic

promoter

A E. coli
pET15N
pET15C
pDest17/17oi
pETG30A
pEGT20A
PKM596
PETG60A

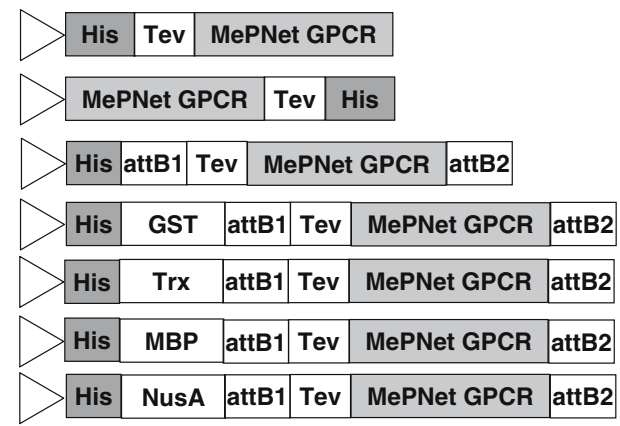

B Pichia pastoris

pPIC9KHisFLAGBiot

\begin{tabular}{|l|l|l|l|l|l|l|}
\hline$\alpha \mathbf{F}$ & FLAG & His & Tev & MePNet GPCR & Tev & Bio \\
\hline
\end{tabular}

C Semliki Forest virus

pSFV2genB

pSFV2genc base without amino acids, 2\% dextrose, $0.00004 \%$ biotin, $1.5 \%$ agar). In a second step the $\mathrm{His}^{+}$clones were cultured on YPD agar containing various concentrations of G418 (1\% yeast extract, $2 \%$ peptone, $2 \%$ dextrose, $2 \%$ agar and $0.05-0.5 \mathrm{mg} / \mathrm{mL}$ G418) to obtain multicopy transformants.

For the production of GPCRs P. pastoris was precultured in BMGY medium (1\% yeast extract, $2 \%$ peptone, $1.34 \%$ yeast nitrogen base without amino acids, $0.00004 \%$ biotin, $1 \%$ glycerol, $0.1 \mathrm{M}$ phosphate buffer, $\mathrm{pH} \mathrm{6)}$ at $30^{\circ} \mathrm{C}$ and $250 \mathrm{rpm}$ until an $\mathrm{OD}_{600}$ value of 2-6 was reached. The induction was carried out in BMMY medium (identical to BMGY except $0.5 \%$ methanol instead of $1 \%$ glycerol) at $30^{\circ} \mathrm{C}$ from an initial $\mathrm{OD}_{600}$ value of 1 . After $18 \mathrm{~h}$ cells were pelleted at $3000 \times g$ for $5 \mathrm{~min}$ and subjected to membrane preparation. Cells were washed once with ice-cold lysis buffer $(50 \mathrm{mM}$ sodium-phosphate buffer pH7.4, $100 \mathrm{mM} \mathrm{NaCl}, 5 \%$ glycerol, $2 \mathrm{mM}$ EDTA, $1 \mathrm{mM}$ PMSF) and resuspended to $30 \%$ wet weight. After $0.5 \mathrm{~mm}$ glass beads were added to the cell suspension, yeast cells were broken by vigorous vortexing at $4^{\circ} \mathrm{C}$ for $10 \mathrm{~min}$. Breaking efficiency was inspected with a light microscope and was usually $>80 \%$. Intact cells and cell debris were separated from the membrane suspension by a low speed centrifugation $\left(3000 \times g, 5 \mathrm{~min}, 4^{\circ} \mathrm{C}\right)$. Membranes were then pelleted using an ultracentrifuge $(100,000 \times g$, $45 \mathrm{~min}, 4^{\circ} \mathrm{C}$ ), resuspended in membrane buffer (50 mM Tris pH8.0, $120 \mathrm{mM} \mathrm{NaCl}, 20 \%$ glycerol, $1 \mathrm{mM}$ PMSF) using a dounce homogenizer. Membrane proteins were quantified applying the BCA method (Pierce, Rockford, IL, USA), using BSA as a standard. Membranes were snap frozen in liquid nitrogen and stored at $-80^{\circ} \mathrm{C}$.

Expression in mammalian cells applying Semliki Forest virus vectors

BHK-21 (baby hamster kidney), CHO-K1 (Chinese hamster ovary) and HEK293 (human embryonic kidney) cells were cultured in a 1:1 mixture of Dulbecco's modified F-12 medium and Iscove's modified Dulbecco's medium supplemented with $4 \mathrm{mM}$ glutamate and 10\% FCS (foetal calf serum). C8166 (human T lymphocyte) cells were grown in RPI Medium, $4 \mathrm{mM}$ glutamate and 10\% FCS. All cell culture reagents were purchased from Gibco BRL (Invitrogen, Carlsbad, CA, USA). Recombinant SFV particles were prepared as previously described (Lundstrom et al. 1994). Briefly, in vitro transcribed RNA from SFV expression vectors pSFV2genB and pSFV2genC, respectively, were co-electroporated with pSFV-Helper2 RNA (carrying the SFV capsid and envelope genes) into BHK-21 cells. Virus was harvested $24 \mathrm{~h}$ later, activated with $\alpha$-chymotrypsin, aliquoted and stored at $-20^{\circ} \mathrm{C}$ (short term) or $-80^{\circ} \mathrm{C}$ (long term). Expression evaluation was performed on 12 and 24 well plates as follows. Adherent cells at $70 \%$ confluency were infected with recombinant SFV particles at various multiplicity of infection (MOI) values and cells harvested at different time points post-infection. Expression evaluation was done on cells lysed in $50 \mathrm{mM}$ Tris-HCl, pH 7.6, $150 \mathrm{mM} \mathrm{NaCl}, 2 \mathrm{mM}$ EDTA, $1 \%$ NP40 and $1 \mu \mathrm{g} / \mu \mathrm{L}$ PMSF. For membrane preparation all manipulations were performed at $4{ }^{\circ} \mathrm{C}$ 
as previously described (Hovius et al. 1998). Approximately $0.5 \mathrm{~g}$ of frozen cell pellets were resuspended in $10 \mathrm{ml}$ of $10 \mathrm{mM}$ HEPES containing $1 \mathrm{mM}$ EDTA (pH 7.4) and homogenized for $10 \mathrm{~s}$ with an UltraTurrax T25 (IKA, Staufen, Germany) homogenizer. Membranes were pelleted by centrifugation at $27,000 \times g$ for $30 \mathrm{~min}$ after a two-fold dilution and resuspended in10 mM HEPES ( $\mathrm{pH} 7.4)$.

\section{Immunodetection methods}

The expression evaluation was visualized by dot blots and/or Western blots. Dot blots. Membranes from $P$. pastoris cells expressing GPCRs were diluted in TBS buffer containing $0.2 \%$ SDS. PVDF membranes (Millipore, $0.45 \mu \mathrm{m}$ ) were washed with methanol and $38 \mathrm{mM}$ Glycine, $10 \mathrm{mM}$ Tris, $20 \%$ methanol in a 96 well microfiltration apparatus (Bio-Dot, BioRad, Reinach, Switzerland) and $1 \mu \mathrm{g}$ of total membrane was added. The PVDF membrane was blocked with $5 \%$ low fat powder in TBS for $60 \mathrm{~min}$ at room temperature, washed three times for 1 min with TBS and incubated with alkaline phosphatase-conjugated anti-FLAG M2 antibody (Sigma, Buchs, Switzerland). After three 15 min washes the blots were developed in AP-buffer (100 mM Tris pH 9.5, $100 \mathrm{mM} \mathrm{NaCl}, 5 \mathrm{mM}$ $\mathrm{MgCl}_{2}$ ) containing $330 \mu \mathrm{g} / \mathrm{mL}$ 5-bromo-4-chloro-indolylphosphate $p$-toluidinium salt and $165 \mu \mathrm{g} / \mathrm{mL}$ nitroblue tetrazolium chloride. For $P$. pastoris membranes, a standard curve for the $\beta 2$ adrenergic receptor corresponding up to $25 \mathrm{pmol}$ receptor per $\mathrm{mg}$ protein, was applied as a reference. In this context, signals of a lower intensity than observed for $1 \mathrm{pmol}$ $\beta 2$ adrenergic receptor were classified as low level (+), intensities corresponding to $1-10 \mathrm{pmol}$ as medium $(++)$ and $>10$ pmol as high $(+++)$. However, these extrapolations are only relative as only part of the signals obtained by immunodetection corresponds to functional receptors. Western blots. Samples were subjected to $10 \%$ SDS-PAGE and the proteins electrotransferred for $30 \mathrm{~min}$ to Hybond ECL nitrocellulose membranes (GE Healthcare, Uppsala, Sweden) and blocked with $5 \%$ milk in TBST (TBS with $0.1 \%$ Tween 20) at $+4^{\circ} \mathrm{C}$. The filter was then treated with primary antibodies (anti-FLAG 1:1000 dilution or anti-His 1:1000 dilution) (Sigma, Buchs, Switzerland), washed, and treated with the secondary anti-mouse antibody 1:2000 dilution (Sigma). The anti-His antibody is directly conjugated to peroxidase and requires no secondary antibody use. The visualization of GPCR-specific bands was done with the ECL chemiluminiscence kit from GE Healthcare (Uppsala, Sweden).
Membrane fractions were prepared from yeast (André et al. 2006) and mammalian (Hovius et al. 1998) cells as previously described. The protein concentration was measured applying the BCA method (Pierce, Rockford, IL, USA) with BSA as a standard. Aliquots of membranes were snap frozen and stored at $-80^{\circ} \mathrm{C}$ prior to use. Radioligand binding assays were established for individual GPCRs as previously described (André et al. 2006 and Hassaine et al. 2006). Briefly, membranes were incubated in triplicates in the presence of radioligand until equilibrium was reached. Non-specific binding was obtained by incubation with excess of non-radioactive ligand. Filtration in presoaked Whatman GF/B or GF/F filters in $0.3 \%$ polyethylenimine was followed by 3 washes and liquid scintillation counting. Single point binding assays were conducted at one non-saturating radioligand concentration. Saturation bindings were performed for certain GPCRs and the saturation curves were analyzed by non-linear regression applying Kaleidagraph (Synergy Software, Reading, PA, USA), which allowed determination of $B_{\max }$ and $K_{\mathrm{d}}$ values.

\section{Results and discussion}

Expression vectors

One hundred and three receptors representing all GPCR families were subcloned after sequence verification into vectors representing the bacterial, yeast and mammalian expression systems of choice. The $\mathrm{N}$-and C-terminally tagged pET15N2 and pET15C2 vectors were used for the expression evaluation of 101 and 18 GPCRs, respectively, and 6 Gateway vectors with or without fusion partners (Fig. 1) were tested for expression of 55 GPCRs in E. coli inclusion bodies. The Pichia pastoris expression vector used in this study has been previously described (André et al. 2006) and was designed on the basis of previous results obtained for GPCR production (Weiss et al. 1998). To facilitate purification efforts and to improve stability of recombinant GPCRs various tags and fusion partners were also introduced into the expression vectors as illustrated in Fig. 1. A signal sequence from the influenza HA (hemagglutinin) gene was further introduced into the SFV vector to promote transport and translocation to the plasma membrane as previously demonstrated for GPCRs expressed in insect cells from baculovirus vectors (Guan et al. 1992). The biotinylation domain from Proprionibacterium shermanii
378

379

380

381

382

383

384

385

386

387

388

389

390

391

392

393

394

395

396

397

398

401

402

403

404

405

406

407

408

409

410

411

412

413

414

415

416

417

418

419

420

421

422

423 
(Bio-tag, Cronan 1990) was engineered into the $P$. pastoris and SFV vectors based on previous experience, which suggested that the biotinylation domain can improve the yields and stability of expressed GPCRs (Weiss et al. 1998, Grünewald et al. 2004, Reinhart and Krettler, 2006). Likewise, the human neurokinin-1 receptor was previously expressed in $\mathrm{SFV}$-infected $\mathrm{BHK}$ and $\mathrm{CHO}$ cells as a fusion protein with the C-terminally tagged biotin (Bio-tag). In this case, the expression levels were monitored by metabolic labeling and saturation binding assays, which indicated similar levels of receptor density for wild type and NK1R-biotin domain constructs (Lundstrom et al. 1995). Furthermore in that study, functional coupling to $\mathrm{G}$ protein was demonstrated by measurement of intracellular $\mathrm{Ca}^{2+}$-release, but no purification was performed to verify the stabilizing effect of the Bio-tag on the GPCR.

\section{Expression evaluation by immunodetection}

Here, the evaluation of expression levels was performed by dot blot and Western blotting techniques and four categories established as follows: high $(+++)$, medium (++), low (+) and no (-) expression. The dot blots were particularly useful to verify the expression levels for individual yeast clones during the selection process, whereas Western blots revealed the size of the expressed GPCR. To qualify as a positive signal in Western blots, the corresponding immunospecific band needed to be in the approximate range of the calculated molecular weight and therefore targets demonstrating bands with significantly faster or slower mobility were considered as negative. The results of the expression evaluation are summarized in Table 1. In E. coli 101 GPCRs were evaluated for expression, of which 46 showed a positive signal by immunoblotting and 55 were negative (Table 1). Eighteen targets belonged to the group of high $(+++), 12$ to medium $(++)$ and 16 to low $(+)$ expression levels. Several factors significantly affected the expressability in bacteria. The choice of $E$. coli vector played a significant role. In this context, the Gateway vectors were superior to the pET15-based vectors. Expression from the N-terminally tagged pET15N2 vector resulted in positive signals in 28 of the 101 tested targets. Introduction of a deca-His tag at the C-terminus in the vector pET15C2 showed positive signals in only 2 of 18 GPCRs tested. In contrast, the success rate was much higher in Gateway vectors, demonstrating positive signals in 33 out of 55 tested targets. Overall, the success of expression of GPCRs in bacteria was relatively low. Characterization of the soluble and the cell pellet fractions in E. coli revealed that the recombinantly expressed GPCRs were uniquely located in inclusion bodies and not inserted into membrane structures, demonstrated by subjecting total bacterial lysates to lysosome treatment and sonication after which inclusion bodies (pellet) were separated from soluble protein (supernatant) by centrifugation and the expressed GPCR verified by SDS-PAGE and Western blotting. Other factors influencing the expression levels were the E. coli strain applied and the growth temperature of bacteria. In this context, the C43 strain was superior to BL1(DE3), Rosetta (DE3) and C41. The initial expression evaluation was carried out at $4 \mathrm{~mL}$ scale. Next, 23 well expressed GPCRs were selected for large-scale production in $1 \mathrm{~L}$ shake flask cultures. Among the 23 GPCRs 9 were expressed from the pET15N2 vector, 1 from pET12C2 and 15 from Gateway vectors. The yields in flask cultures varied between $2 \mathrm{mg} / \mathrm{L}$ and $40 \mathrm{mg} / \mathrm{L}$ and the recombinant receptors were only localized in inclusion bodies. Although the yields were relatively high the produced material was not sufficient for extensive purification and refolding exercises. For this reason, 7 of the 23 targets were further subjected to fermentation in 3 or $8 \mathrm{~L}$ bioreactors. The yields in the fermentor cultures were generally higher $(75-350 \mathrm{mg} / \mathrm{L})$ than in flask cultures and provided now sufficient material for the establishment of purification and refolding conditions.

Only a single vector construct was engineered for P. pastoris (Fig. 1B). Briefly, the vector contained an inducible AOX1 promoter followed by an $\alpha$-factor signal sequence and FLAG- and deca-His-tags at the $\mathrm{N}$-terminus. The GPCRs were introduced as BamHISpeI fragments and flanked by Tev protease cleavage sites. These sites were engineered into the expression vector to allow removal of all tags after purification to avoid any interference of tags during the crystallization procedure. Furthermore, the Bio-tag was engineered at the C-terminal to potentially improve expression and stability of the recombinant GPCRs (Reinhart and Krettler 2006). After subcloning of the GPCRs into the $P$. pastoris vector pPIC9K, a high-throughput screening method based on in situ immunoblotting was established (Magnin et al. manuscript in preparation). This method facilitated significantly the clone selection process of the large number of GPCR constructs studied in parallel. The expression profile in yeast cells applying $P$. pastoris vectors was quite different from what was obtained in bacteria. The success rate was much higher, partly due to the clone selection process for each individual GPCR, which was established to obtain constructs with optimum number of copies integrated into the yeast genome (André et al. 2006). 
Table 1 Expression evaluation by immunodetection

\begin{tabular}{|c|c|c|c|c|c|c|}
\hline & MePNet\# & E. coli & P. pastoris & SFV & Size & $\mathrm{GC} \%$ \\
\hline ACM1_HUMAN & 23 & - & + & +++ & 51.4 & 61 \\
\hline ACM1_MOUSE & 24 & - & + & +++ & 51.3 & 57 \\
\hline ACM2_PIG & 26 & - & + & + & 51.7 & 47 \\
\hline ACM2_HUMAN & 25 & - & + & + & 51.7 & 47 \\
\hline A1AA_HUMAN & 104 & - & + & +++ & 51.5 & 57 \\
\hline A1AA_RAT & 14 & - & + & +++ & 51.6 & 55 \\
\hline AA1B_HUMAN & 15 & nd & ++ & ++ & 56.8 & 63 \\
\hline AA1B_MESAU & 105 & - & + & + & 56.5 & 60 \\
\hline A1AD_RAT & 106 & - & + & + & 59.4 & 62 \\
\hline A2AA_HUMAN & 107 & - & + & +++ & 49 & 69 \\
\hline A2AB_HUMAN & 16 & - & ++ & + & 50 & 64 \\
\hline A2AC_HUMAN & 18 & - & ++ & + & 49.6 & 66 \\
\hline B3AR_HUMAN & 31 & - & + & + & 43.5 & 70 \\
\hline D2DR_HUMAN & 43 & - & ++ & ++ & 50.6 & 57 \\
\hline D2DR_MOUSE & 44 & - & +++ & +++ & 50.9 & 53 \\
\hline D3DR_HUMAN & 45 & + & ++ & +++ & 44.2 & 55 \\
\hline D4DR_HUMAN & 46 & + & ++ & & 48.4 & 75 \\
\hline DADR_HUMAN & 109 & - & ++ & ++ & 49.3 & 52 \\
\hline DBDR_HUMAN & 110 & - & ++ & +++ & 53 & 61 \\
\hline HH2R_HUMAN & 61 & + & +++ & +++ & 40.1 & 57 \\
\hline HH2_RAT & 62 & + & nd & +++ & 40.3 & 54 \\
\hline 5H1A_HUMAN & 1 & - & +++ & +++ & 46.1 & 60 \\
\hline 5H1B_HUMAN & 2 & + & +++ & +++ & 43.6 & 57 \\
\hline 5H1D_HUMAN & 3 & - & +++ & +++ & 41.9 & 56 \\
\hline 5H1E_HUMAN & 4 & - & ++ & + & 41.7 & 51 \\
\hline 5H1F_HUMAN & 5 & - & +++ & +++ & 41.7 & 41 \\
\hline 5H2A_HUMAN & 6 & ++ & ++ & + & 52.6 & 46 \\
\hline 5H2A_MOUSE & 7 & ++ & & + & 52.8 & 48 \\
\hline 5H2B_HUMAN & 8 & - & + & + & 54.3 & 43 \\
\hline 5H2C_HUMAN & 9 & - & - & +++ & 51.8 & 45 \\
\hline 5H4_HUMAN & 144 & - & ++ & +++ & 43.8 & 48 \\
\hline 5H5A_HUMAN & 10 & - & ++ & +++ & 40.3 & 61 \\
\hline 5H6_RAT & 111 & - & ++ & +++ & 46.9 & 64 \\
\hline 5H7_HUMAN & 12 & + & + & +++ & 53.6 & 56 \\
\hline 5H7_RAT & 117 & ++ & ++ & - & 49.8 & 56 \\
\hline AG2R_HUMAN & 28 & ++ & ++ & + & 41.1 & 40 \\
\hline BRB1_HUMAN & 32 & & + & +++ & 40.4 & 55 \\
\hline BRB2_HUMAN & 33 & 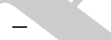 & + & +++ & 44.5 & 58 \\
\hline FSHR_HUMAN & 131 & - & + & + & 78.3 & 59 \\
\hline IL8A_HUMAN & 63 & + & + & ++ & 39.8 & 53 \\
\hline IL8B_HUMAN & 136 & - & + & + & 40.8 & 53 \\
\hline CCR3_HUMAN & 39 & -1 & + & +++ & 40.7 & 64 \\
\hline CCR4_HUMAN & 135 & nd & nd & +++ & 39.7 & 50 \\
\hline CKR1_HUMAN & 40 & + & +++ & +++ & 41.1 & 50 \\
\hline CKR2_HUMAN & 41 & + & nd & + & 41.9 & 47 \\
\hline CKR6_HUMAN & 42 & +++ & + & ++ & 42.5 & 47 \\
\hline CKR7_HUMAN & 145 & + & + & ++ & 42.9 & 56 \\
\hline CKRB_HUMAN & 153 & - & + & +++ & 39.9 & 41 \\
\hline CCKR_HUMAN & 38 & - & +++ & ++ & 47.8 & 54 \\
\hline GASR_HUMAN & 57 & - & +++ & ++ & 48.4 & 63 \\
\hline ET1R_BOVIN & 50 & +++ & +++ & - & 48.5 & 47 \\
\hline ET1R_HUMAN & 51 & +++ & + & + & 48.7 & 45 \\
\hline ACTR_HUMAN & 27 & +++ & ++ & + & 33.9 & 51 \\
\hline ETBR_BOVIN & 52 & + & ++ & + & 49.4 & 49 \\
\hline MC3R_HUMAN & 64 & ++ & +++ & + & 40 & 57 \\
\hline MC4R_HUMAN & 65 & +++ & +++ & + & 37 & 46 \\
\hline MC5R_HUMAN & 66 & ++ & +++ & + & 36.6 & 53 \\
\hline NY1R_HUMAN & 79 & +++ & +++ & +++ & 44.4 & 40 \\
\hline NY2R_HUMAN & 80 & - & +++ & ++ & 42.7 & 50 \\
\hline NY4R_HUMAN & 81 & ++ & ++ & ++ & 42.2 & 57 \\
\hline NY5R_HUMAN & 82 & - & + & nd & 52 & 38 \\
\hline OPRD_HUMAN & 83 & - & + & + & 40.4 & 65 \\
\hline
\end{tabular}


Table 1 continued

\begin{tabular}{|c|c|c|c|c|c|c|}
\hline & MePNet\# & E. coli & P. pastoris & SFV & Size & $\mathrm{GC} \%$ \\
\hline OPRD_MOUSE & 84 & - & ++ & ++ & 40.6 & 63 \\
\hline OPRK_HUMAN & 85 & +++ & ++ & ++ & 42.7 & 54 \\
\hline OPRK_MOUSE & 86 & +++ & +++ & + & 42.7 & 49 \\
\hline SSR1_HUMAN & 93 & - & +++ & +++ & 42.7 & 61 \\
\hline SSR2_HUMAN & 94 & +++ & ++ & +++ & 41.3 & 52 \\
\hline SSR3_HUMAN & 95 & - & +++ & +++ & 45.8 & 66 \\
\hline NK1R_HUMAN & 72 & +++ & ++ & +++ & 46.3 & 55 \\
\hline NK1R_RAT & 73 & + & +++ & + & 46.4 & 55 \\
\hline NK2R_HUMAN & 74 & +++ & ++ & +++ & 44.4 & 58 \\
\hline NK2R_RAT & 75 & ++ & +++ & +++ & 43.9 & 57 \\
\hline NK3R_HUMAN & 76 & +++ & +++ & ++ & 52.2 & 51 \\
\hline V1BR_HUMAN & 103 & ++ & +++ & +++ & 47 & 63 \\
\hline GALR_HUMAN & 54 & - & ++ & +++ & 38.9 & 58 \\
\hline GALT_HUMAN & 56 & +++ & +++ & ++ & 39.6 & 72 \\
\hline PAR1_HUMAN & 99 & - & + & - & 47.4 & 50 \\
\hline MCR1_HUMAN & 60 & - & +++ & ++ & 46 & 57 \\
\hline LSHR_RAT & 133 & - & - & +++ & 78 & 49 \\
\hline FML1_HUMAN & 139 & - & ++ & ++ & 39 & 51 \\
\hline TSHR_RAT & 116 & - & + & + & 86.5 & 50 \\
\hline PE23_HUMAN & 90 & ++ & + & ++ & 43.3 & 60 \\
\hline AA1R_HUMAN & 19 & + & + & ++ & 36.5 & 60 \\
\hline AA2A_HUMAN & 20 & ++ & + & ++ & 44.7 & 61 \\
\hline AA2B_HUMAN & 21 & +++ & + & ++ & 36.3 & 55 \\
\hline P2Y2_MOUSE & 87 & - & - & + & 42.2 & 63 \\
\hline P2Y2_HUMAN & 88 & - & - & +++ & 39.4 & 36 \\
\hline P2Y6_HUMAN & 137 & - & ++ & + & 36.4 & 63 \\
\hline P2YR_HUMAN & 89 & +++ & +4 & +++ & 42.1 & 51 \\
\hline CB1R_HUMAN & 36 & nd & + & nd & 52.9 & 54 \\
\hline CB1R_MOUSE & 121 & +++ & + & + & 52.8 & 52 \\
\hline CB2R_HUMAN & 37 & + & +++ & +++ & 39.7 & 56 \\
\hline EDG3_HUMAN & 48 & ++ & 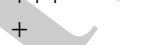 & +++ & 42.3 & 58 \\
\hline CLT1_HUMAN & 129 & - & - & +++ & 38.5 & 39 \\
\hline CLT2_HUMAN & 130 & +++ & ++ & + & 39.6 & 45 \\
\hline CALR_HUMAN & 34 & - & + & - & 57.3 & 47 \\
\hline GLR_HUMAN & 59 & & + & ++ & 54 & 63 \\
\hline MGR2_HUMAN & 67 & & + & - & 95.5 & 61 \\
\hline MGR3_HUMAN & 68 & & +++ & + & 98.6 & 51 \\
\hline MGR4_HUMAN & 69 & & +++ & +++ & 101.9 & 61 \\
\hline MGR5_HUMAN & 113 & & nd & nd & 126.7 & 61 \\
\hline MGR7_HUMAN & 114 & . & + & + & 102.3 & 51 \\
\hline STE2_YEAST & 96 & + & + & +++ & 47.8 & 38 \\
\hline STE3_YEAST & 97 & - & +++ & +++ & 53.7 & 38 \\
\hline
\end{tabular}

nd, not done; no expression, - (pink); low expression, + (light blue); medium expression, ++ (medium blue); high expression, +++ (dark blue)

Additionally, the eukaryotic status of yeast cells most likely contributed to the success in expression. Overall, 94 of 100 evaluated GPCRs showed a positive signal by immunodetection, of which 30 belonged to the group of high $(+++), 27$ to medium $(++)$ and 37 to low $(+)$ Table 1$)$. Only 6 GPCRs showed no expression in $P$. pastoris. The expression pattern in yeast was also strongly affected by external factors. The cell culture temperature, addition of ligands and additive supplements to the yeast culture medium strongly influenced the expression levels. In this context, the lowering of the temperature from $30^{\circ} \mathrm{C}$ to $20^{\circ} \mathrm{C}$, addition of specific ligand at concentrations close to 100 times the $K_{\mathrm{d}}$ value or providing additives such as $2.5 \%$ DMSO or $0.04 \mathrm{mg}$ / $\mathrm{mL}$ histidine significantly increased the binding activity (André et al. 2006). The results, however, varied considerably from one GPCR to another. For instance, lowering of the temperature improved the binding activity for 10 GPCRs, had no significant effect on 9 receptors and for the human serotonin 5-HT1B receptor resulted in substantially reduced activity. Likewise, treatment with DMSO resulted in increased binding for 16 GPCRs whereas 4 receptors were unaffected. Addition of ligand also improved the binding activity for 18 receptors, but reduced $B_{\max }$ values were observed for the human serotonin 5-HT1B 
and the rat neurokinin- 2 receptors. The effect of histidine addition was favorable for 12 GPCRs and indifferent for 8 . To further optimize the expression those parameters with a positive effect were combined, which resulted in doubled production levels for 13 GPCRs. The $B_{\max }$ values were more than 4 times higher for 5 receptors and the maximum increase of 8.7 fold was obtained for the human kappa opioid receptor. Interestingly, when the expression levels in Dot blots were compared to the $B_{\max }$ values, it was evident that optimization did not affect the receptor quantity (Fig. 2). It rather looked like the quality of the recombinant GPCRs was enhanced resulting in larger proportion of receptors with functional binding activity. The optimal expression conditions for individual GPCRs have been described in more detail previously (André et al. 2006).

Two expression vectors were designed for SFVbased expression (Fig. 1C). In the pSFV2genB vector a Kozak sequence was introduced downstream of the subgenomic SFV 26S promoter to optimize the initiation of ribosomes (Kozak 1986). A signal sequence from the influenza hemagglutinin (HA) gene was introduced to facilitate the translocation of the GPCRs to the plasma membrane. Furthermore, a FLAG tag was engineered at the $\mathrm{N}$-terminus in front of the fulllength GPCR flanked by Tev protease cleavage sites. The C-terminal contained a deca-His tag. The second SFV vector, pSFV2genC, was otherwise identical to pSFV2genB except for an additional biotin tag placed downstream of the His-10 tag. One hundred and one GPCRs were evaluated for expression in mammalian cells using SFV-infected host cells. Due to the broad host range of SFV, several mammalian cell lines can be efficiently transduced. However, dealing with such a
A

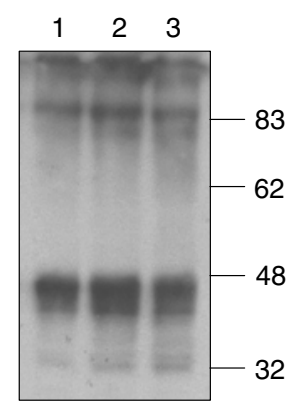

B
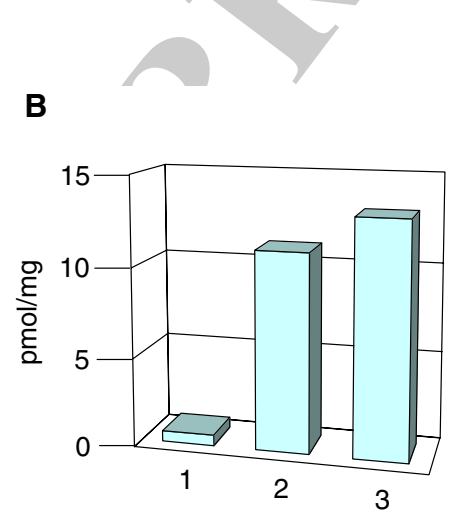

Fig. 2 Expression of the human NK2 receptor in $P$. pastoris: comparison of Western blots to binding activity before and after optimization. Sample $1,30^{\circ} \mathrm{C}$; sample $2,20^{\circ} \mathrm{C}$; sample $3,20^{\circ} \mathrm{C}+$ 2.5\% DMSO. (A) Western blots using anti-FLAG M2 antibody. Molecular weight marker indicated on the right. (B) Saturation binding using $\left[{ }^{3} \mathrm{H}\right] \mathrm{SR} 48968$ large number of targets, it was decided to initially limit the expression evaluation to three cell lines, BHK-21 (baby hamster kidney), CHO-K1 (Chinese hamster ovary) and HEK293 (human embryonic kidney) cells, previously shown to express high levels of GPCRs (Lundstrom 2003). A few GPCRs were also expressed in the human T lymphocyte cell line C8166. The success rate for SFV expression was very high with 97 GPCRs showing positive signals and only 4 targets no detectable specific response in Western blots (Table 1). According to expression evaluation 44 GPCRs were classified as high $(+++), 25$ as medium $(++)$ and 28 as low $(+)$ expressers. Important factors in relation to expression levels were virus concentration (MOI, multiplicity of infection), and the duration of expression and host cell line. Taken together the expression results from the three systems demonstrated that positive signals were obtained for 102 GPCRs verified by immunodetection, of which 65 represented high $(+++), 20$ medium $(++)$ and 19 low $(+)$ expression levels. Only one GPCR (MGR5_HUMAN) was negative, although only tested for expression in E. coli. In fact, the human metabotropic glutamate mGluR5 has been previously successfully expressed from SFV vectors (Lundstrom 2000). Likewise, the glutamate receptor mGluR2 (Schweitzer et al. 2000) and the endothelin receptor (Cramer et al. 2001), both negative in this study when expressed from SFV vectors, demonstrated high specific binding and functional activity in mammalian cells. This discrepancy may be due to the use of the standard SFV vector in the previous studies, whereas here the vector contained the influenza HA signal sequence, which might interfere with the expression, folding and transport of the recombinant receptor.

Expression comparison of GPCR families and subtypes

The comparison of expressability of GPCRs from the four major classes, A rhodopsin-like, B secretin-like, C metabotropic glutamate/pheromone and D fungal pheromone is not possible as the majority of GPCRs studied are class A receptors (95) and only very few representatives were from the other classes: B (2), C (4) and D (2). The reason for having such a high representation of class A receptors is their large number and their importance as drug targets. The expression pattern for the class A receptors showed a success rate of $48.4 \%$ in E. coli, $93.5 \%$ in P. pastoris and $96.8 \%$ in SFV evaluated by immunodetection. The expression pattern for the bacterial expression of the few targets of the other classes of GPCRs was similar except for 
640

641

642

643

644

645

646

647

648

649

650

651

652

653

654

655

656

657

658

659

660

661

662

663

664

665

666

667

668

669

670

671

672

673

674

675

676

677

678

679

680

681

682

683

684

685

686

687

688

689

690 the class $\mathrm{C}$ receptors, where none of the four tested GPCRs gave a signal. Class $\mathrm{C}$ receptors in general and the four metabotropic glutamate receptors tested here are characterized by a large extracellular $\mathrm{N}$-terminal domain. Whether the extracellular domain or the large size of the recombinant metabotropic glutamate receptors $(95-102 \mathrm{kD})$ was the reason for failure of expression is discussed below under target size. The yeast expression showed $100 \%$ success rate for class B, $\mathrm{C}$ and D receptors and for SFV-based expression in mammalian cells most of the GPCRs were positive by immunodetection.

More interestingly, among the GPCRs studied several belonged to the same subfamily of GPCRs. For instance, 4 muscarinic, $4 \alpha$-adrenergic, 6 dopamine, 14 serotonin, 4 neuropeptide Y, 4 opioid, 5 neurokinin, 4 purinoreceptor $\mathrm{P} 2 \mathrm{Y}$ and 4 metabotropic glutamate receptors were studied (Table 2). The expressability of the GPCRs was evaluated by immunodetection and grouped as previously into categories of no expression $(-)$, low expression $(+)$, medium expression $(++)$ and high expression $(+++)$. The serotonin receptors, the group with the highest number of subtypes, demonstrated no expression for $64.2 \%$ of the targets in $E$. coli, medium expression for $42.9 \%$ in $P$. pastoris and high expression of $57.1 \%$ in SFV. Overall, high expression levels were obtained for $31.0 \%$ of the serotonin receptors. Another subfamily of receptors that was well expressed for all three expression systems, were the neurokinin receptors. Of the 5 neurokinin receptors tested $60 \%$ were expressed at high levels in all three expression systems. None of the neurokinin receptors showed a negative signal. The 8 adrenergic receptors showed variable expression levels. None of them were successfully expressed in $E$. coli, the expression levels in yeast were either low or medium, whereas in mammalian cells 4 GPCRs showed low, 1 target medium and 3 targets high expression levels. Dopamine receptors were also problematic to express in E. coli as all 6 subtypes were negative. In $P$. pastoris the majority of dopamine receptors were expressed at medium levels, whereas for SFV 4 of the 6 GPCRs belonged to the group of high expressers.

\section{Target size and GC content}

The size of recombinantly expressed proteins might play an important role in relation to the expression levels obtained. In general, smaller proteins have given better expression yields especially in bacterial systems. In the case of the 100 targets studied here, they represented GPCRs ranging in size from a postulated molecular weight of $33.9 \mathrm{kD}$ to $102.3 \mathrm{kD}$. Comparison of molecular weight to expression levels suggested that size played an important role in bacterial expression. In this context, the largest class A GPCR expressed successfully from $E$. coli had a postulated molecular weight of $53.6 \mathrm{kD}$. Similarly, the class B receptor with a size of $54.0 \mathrm{kD}$ gave a positive signal whereas the GPCR of $57.3 \mathrm{kD}$ was negative in Western blots. All 4 class C GPCRs were negative in E. coli. These metabotropic glutamate receptor are large (95-102 kD) and it is therefore not surprising that no expression was observed. Of the two class D yeast STE receptors, only the smaller STE2 $(47.8 \mathrm{kD})$ was expressed, whereas the STE3 $(53.7 \mathrm{kD})$ showed no signal. According to the expression results for the 93 class A GPCRs expressed in E. coli, $54 \mathrm{kD}$ seemed to be the limit where positive signals were detected. In contrast to bacterial expression, when the 100 GPCRs were analyzed for expression in yeast and mammalian cells, no correlation could be observed between size and expressability. For example, all of the large size class $C$ receptors were expressed in $P$. pastoris and 3 out 4 of them expressed in SFV.

Among factors influencing gene expression in general, the different codon usage in pro- and eukaryotes has been indicated as a cause. This issue has been addressed by developing special bacterial strains for recombinant proteins with poor codon usage for mammalian proteins (Kleber-Janke and Becker 2000). It has also been shown that the frequency of some codons and amino acids correlate with the GC content in the genome (Sueoka 1961). Recently, the trends in codon and amino acid usage in relation to GC composition have been studied (Knight et al. 2001). Here we have investigated whether the GC content has any effect on the expression levels of 103 GPCRs in bacterial, yeast and mammalian cells. The GC content for the 103 GPCRs varied between $36 \%$ and $75 \%$ for class A receptors. The two class B GPCRs had GC contents of $47 \%$ and $63 \%$, respectively. Class $\mathrm{C}$ receptors showed a range of GC content from $51 \%$ to $61 \%$ and the two class D receptors had both a GC content of $38 \%$. In none of the three expression systems was there any correlation between the expression levels and the GC content. Also the GPCRs that showed no signal showed a large variation in GC content.

Immunoblotting versus binding activity

GPCRs expressed in E. coli inclusion bodies are present in aggregates and require refolding before any functional activity can be restored. In contrast, expression of GPCRs in yeast and mammalian cells 
Table 2 Expression comparison of GPCR subtypes.

\begin{tabular}{|c|c|c|c|c|c|}
\hline $\mathrm{Nd}$ & - & + & ++ & +++ & \\
\hline \multicolumn{6}{|c|}{ Muscarinic (4) } \\
\hline 0 & 4 / 100\% & 0 & 0 & 0 & E. coli \\
\hline 0 & 0 & $4 / 100 \%$ & 0 & 0 & P. pastoris \\
\hline 0 & 0 & $2 / 50.0 \%$ & 0 & $2 / 50.0 \%$ & SFV \\
\hline 0 & $4 / 33.3 \%$ & $6 / 50.0 \%$ & 0 & $2 / 16.7 \%$ & Total \\
\hline \multicolumn{6}{|c|}{ Adrenergic (8) } \\
\hline 1 & $7 / 100 \%$ & 0 & 0 & 0 & E. coli \\
\hline 0 & 0 & $5 / 62.5 \%$ & $3 / 37.5 \%$ & 0 & P. pastoris \\
\hline 0 & 0 & $4 / 50 \%$ & $1 / 12.5 \%$ & $3 / 37.5 \%$ & SFV \\
\hline 1 & $7 / 30.4 \%$ & $9 / 39.1 \%$ & $4 / 17.4 \%$ & $3 / 13.0 \%$ & Total \\
\hline \multicolumn{6}{|c|}{ Dopamine (6) } \\
\hline 0 & 4 / 100\% & 0 & 0 & 0 & E. coli \\
\hline 0 & 0 & 0 & $5 / 83.3 \%$ & $1 / 16.7 \%$ & P. pastoris \\
\hline 0 & 0 & 0 & $2 / 33.3 \%$ & $4 / 66.7 \%$ & SFV \\
\hline 0 & $4 / 22.2 \%$ & 0 & $7 / 38.9 \%$ & $5 / 27.8 \%$ & Total \\
\hline \multicolumn{6}{|c|}{ Serotonin (14) } \\
\hline 0 & $9 / 64.2 \%$ & $2 / 14.3 \%$ & $2 / 14.3 \%$ & $1 / 7.1 \%$ & E. coli \\
\hline 0 & $2 / 14.3 \%$ & $2 / 14.3 \%$ & $6 / 42.9 \%$ & $4 / 28.6 \%$ & P. pastoris \\
\hline 0 & 0 & $3 / 21.4 \%$ & $2 / 14.3 \%$ & $9 / 64.3 \%$ & SFV \\
\hline 0 & $11 / 26.2 \%$ & $7 / 16.7 \%$ & $10 / 23.8 \%$ & $14 / 33.3 \%$ & Total \\
\hline \multicolumn{6}{|c|}{ Neuropeptide $Y$ (4) } \\
\hline 0 & $2 / 50.0 \%$ & 0 & $1 / 25.0 \%$ & $1 / 25.0 \%$ & E. coli \\
\hline 0 & 0 & $1 / 25.0 \%$ & $1 / 25.0 \%$ & $2 / 50.0 \%$ & P. pastoris \\
\hline 1 & 0 & 0 & $2 / 66.7 \%$ & $1 / 33.3 \%$ & SFV \\
\hline 1 & $2 / 18.2 \%$ & $1 / 9.1 \%$ & $4 / 36.4 \%$ & $4 / 36.4 \%$ & Total \\
\hline \multicolumn{6}{|c|}{ Opioid (4) } \\
\hline 0 & $2 / 50.0 \%$ & 0 & 0 & $2 / 50.0 \%$ & E. coli \\
\hline 0 & 0 & $1 / 25.0 \%$ & $2 / 50.0 \%$ & $1 / 25.0 \%$ & P. pastoris \\
\hline 0 & 0 & $1 / 50.0 \%$ & $3 / 50.0 \%$ & 0 & SFV \\
\hline 0 & $2 / 16.7 \%$ & $2 / 16.7 \%$ & $5 / 41.7 \%$ & $3 / 25.0 \%$ & Total \\
\hline \multicolumn{6}{|c|}{ Neurokinin (5) } \\
\hline 0 & 0 & $1 / 20 \%$ & $1 / 20 \%$ & $3 / 60.0 \%$ & E. coli \\
\hline 0 & 0 & 0 & $2 / 40 \%$ & $3 / 60.0 \%$ & P. pastoris \\
\hline 0 & 0 & $1 / 20 \%$ & $1 / 20 \%$ & $3 / 60.0 \%$ & SFV \\
\hline 0 & $0.00 \%$ & $2 / 13.3 \%$ & $4 / 26.7 \%$ & $9 / 60.0 \%$ & Total \\
\hline \multicolumn{6}{|c|}{ Purinoreceptor $P 2 Y$ (4) } \\
\hline 0 & $3 / 75.0 \%$ & 0 & 0 & $1 / 25.0 \%$ & E. coli \\
\hline 0 & $2 / 50.0 \%$ & 0 & $1 / 25.0 \%$ & $1 / 25.0 \%$ & P. pastoris \\
\hline 0 & 0 & $2 / 50.0 \%$ & 0 & $2 / 50.0 \%$ & SFV \\
\hline 0 & $5 / 41.7 \%$ & $2 / 16.7 \%$ & $1 / 8.3 \&$ & $4 / 33.3 \%$ & Total \\
\hline \multicolumn{6}{|c|}{ Metabotropic glutamate (5) } \\
\hline 0 & $5 / 100 \%$ & 0 & 0 & 0 & E. coli \\
\hline 1 & 0 & $2 / 50.0 \%$ & 0 & $2 / 50.0 \%$ & P. pastoris \\
\hline 1 & $1 / 20.0 \%$ & $1 / 20.0 \%$ & $1 / 20.0 \%$ & $1 / 20.0 \%$ & SFV \\
\hline 2 & $6 / 40.0 \%$ & $3 / 20.0 \%$ & $1 / 6.7 \%$ & $3 / 20.0 \%$ & Total \\
\hline
\end{tabular}

Expression levels as for Table 1; highlighted in yellow; the highest number of targets expressed from individual expression vectors and in total

generate receptors located in cell membranes, which can be directly monitored for their functional activity by radioligand binding assays. Selected GPCRs were subjected to binding assays and the correlation between immunodetection and binding activity was evaluated (Table 3). A total of 51 GPCRs expressed in $P$. pastoris and 42 in SFV were assayed for specific binding. The GPCRs were divided into four groups indicating no $(0)$, low $(<1 \mathrm{pmol} / \mathrm{mg})$, medium (1-10 pmol/mg) and high specific binding (> $10 \mathrm{pmol} /$ mg). Expression in yeast cells generated 7 targets with no, 15 with low, 13 with medium and 16 with high specific binding activity. The highest $B_{\max }$ value of $180 \mathrm{pmol} / \mathrm{mg}$ was obtained for the adenosine A2A receptor (AA2A_HUMAN). For SFV-based expression, 3 targets showed no, 7 low, 18 medium and 14 high specific binding. Again, the highest binding activity (287 pmol/mg) was obtained for AA2A_HUMAN. The correlation between immunodetection and specific binding activity was analyzed as shown in 
Table 3 Immunodetection vs. binding activity of selected GPCRs

\begin{tabular}{|c|c|c|c|c|c|}
\hline & MePNet\# & Pichia IB & Pichia B & SFV ID & SFV BA \\
\hline ACM1_HUMAN & 23 & + & 0.23 & +++ & 2.44 \\
\hline ACM1_MOUSE & 24 & + & 1 & +++ & 2.93 \\
\hline ACM2_PIG & 26 & + & 1 & + & 22 \\
\hline ACM2_HUMAN & 25 & + & 1.5 & + & 6.1 \\
\hline A1AA_HUMAN & 104 & + & 0.45 & +++ & 0.25 \\
\hline A1AA_RAT & 14 & + & 0.25 & +++ & 4.7 \\
\hline AA1B_HUMAN & 15 & ++ & 5 & ++ & 0 \\
\hline AA1B_MESAU & 105 & + & 0.14 & + & 0 \\
\hline A1AD_RAT & 106 & + & 0.21 & + & 0 \\
\hline A2AB_HUMAN & 16 & ++ & 11 & + & 0.05 \\
\hline A2AC_HUMAN & 18 & ++ & 0.25 & + & 0.65 \\
\hline B3AR_HUMAN & 31 & + & 0 & + & nd \\
\hline D2DR_HUMAN & 43 & ++ & 39 & ++ & 29.2 \\
\hline D2DR_MOUSE & 44 & +++ & 65 & +++ & 21.8 \\
\hline D3DR_HUMAN & 45 & ++ & 1.7 & +++ & 1.8 \\
\hline D4DR_HUMAN & 46 & ++ & 2 & +++ & 3.47 \\
\hline DBDR_HUMAN & 110 & ++ & 0 & +++ & nd \\
\hline HH2R_HUMAN & 61 & +++ & 50 & +++ & 20 \\
\hline 5H1A_HUMAN & 1 & +++ & 0.9 & +++ & 12.7 \\
\hline 5H1B_HUMAN & 2 & +++ & 38 & +++ & 19.2 \\
\hline 5H1D_HUMAN & 3 & +++ & 102 & +++ & 11 \\
\hline 5H1E_HUMAN & 4 & ++ & 0.8 & + & 0.75 \\
\hline 5H1F_HUMAN & 5 & +++ & 0 & +++ & 0.69 \\
\hline 5H2A_HUMAN & 6 & ++ & 1.8 & ++ & 8.5 \\
\hline 5H5A_HUMAN & 10 & ++ & 24 & +++ & 31 \\
\hline 5H6_RAT & 111 & ++ & 1 & +++ & 1.4 \\
\hline 5H7_HUMAN & 12 & + & 11.8 & +++ & 2.2 \\
\hline 5H7_RAT & 117 & ++ & 1.5 & - & 2.18 \\
\hline AG2R_HUMAN & 28 & ++ & 0 & + & nd \\
\hline BRB1_HUMAN & 32 & + & 0.11 & +++ & 0.19 \\
\hline BRB2_HUMAN & 33 & + & 0.13 & +++ & 7.9 \\
\hline ET1R_BOVIN & 50 & ++ & 3.5 & - & nd \\
\hline ET1R_HUMAN & 51 & + & 0.1 & + & nd \\
\hline ETBR_BOVIN & 52 & ++ & 0.4 & + & nd \\
\hline NY1R_HUMAN & 79 & +++ & 60 & +++ & 2.3 \\
\hline NY2R_HUMAN & 80 & +++ & 0 & + & 1.85 \\
\hline OPRD_HUMAN & 83 & & 3 & + & 1.85 \\
\hline OPRD_MOUSE & 84 & & 3.7 & ++ & 4 \\
\hline OPRK_HUMAN & 85 & ++ & 25 & ++ & 1.6 \\
\hline OPRK_MOUSE & 86 & +++ & 45 & ++ & 1 \\
\hline SSR1_HUMAN & 93 & +++ & 0.1 & +++ & nd \\
\hline SSR3_HUMAN & 95 & +++ & 0 & +++ & nd \\
\hline NK1R_HUMAN & 72 & ++ & 15 & +++ & 56.7 \\
\hline NK1R_RAT & 73 & +++ & 1.9 & + & 68.9 \\
\hline NK2R_HUMAN & 74 & ++ & 39 & +++ & 3.6 \\
\hline NK2R_RAT & 75 & +++ & 162 & +++ & 46.4 \\
\hline NK3R_HUMAN & 76 & +++ & 23 & ++ & 26.5 \\
\hline V1BR_HUMAN & 103 & +++ & 0 & +++ & 26.1 \\
\hline MCR1_HUMAN & 60 & +++ & 0.01 & nd & nd \\
\hline AA1R_HUMAN & 19 & + & 0.8 & ++ & 0.4 \\
\hline AA2A_HUMAN & 20 & + & 180 & ++ & 287 \\
\hline
\end{tabular}

Expression levels as for Table 1

762 Table 4. This comparison could obviously only be performed for GPCRs expressed in yeast and mammalian cells as the E. coli expressed GPCRs were located in inclusion bodies and required refolding to regain functional binding activity. In this case, there seemed to be no evident correlation between the intensity of the signals obtained in immunoblots and/or Western blots and the specific binding in agreement with previous observations (André et al. 2006). More than half $(56.3 \%)$ of those targets that showed low expression levels by immunodetection also produced specific binding below $1 \mathrm{pmol} / \mathrm{mg}$. $38.9 \%$ of the 
Table 4 Correlation between immunodetection and specific binding

\begin{tabular}{llll}
\hline & + & ++ & ++ \\
\hline$P$. pastoris & 16 & 18 & 17 \\
0 & $1 / 6.3 \%$ & $2 / 11.1 \%$ & $4 / 23.5 \%$ \\
$<1$ pmol & $9 / 56.3 \%$ & $7 / 16.7 \%$ & $2 / 17.6 \%$ \\
$1-10$ pmol & $4 / 25 \%$ & $6 / 33.9 \%$ & $8 / 47.1 \%$ \\
$>10$ pmol & $2 / 12.5 \%$ & 10 & 25 \\
SFV & 14 & $1 / 10.0 \%$ & $0 / 0 \%$ \\
0 & $2 / 16.7 \%$ & $1 / 10.0 \%$ & $2 / 8.0 \%$ \\
$<1$ pmol & $3 / 21.4 \%$ & $4 / 40.0 \%$ & $11 / 44.0 \%$ \\
$1-10$ pmol & $3 / 21.4 \%$ & $3 / 30.0 \%$ & $11 / 44.0 \%$ \\
$>10$ pmol & $2 / 14.3 \%$ & $1 / 10.0 \%$ & $3 / 12 \%$ \\
nd & $4 / 28.6 \%$ & $12 \%$ & \\
\hline
\end{tabular}

Expression levels as for Table 1; highlighted in yellow; the highest number of targets expressed from individual expression vectors and in total

GPCRs demonstrating medium expression levels also showed medium binding activity (1-10 pmol/mg) although almost the same percentage was achieved for binding over $10 \mathrm{pmol} / \mathrm{mg} \quad(33.3 \%)$. Almost half $(47.1 \%)$ of the GPCRs which showed high expression levels by immunodetection also belonged to the group of targets with high binding activity. The situation for SFV-expressed targets was similar. Among the GPCRs with low expression levels in Western blots also $21.4 \%$ showed binding activities below $1 \mathrm{pmol} / \mathrm{mg}, 41.7 \%$ of this group produced specific binding values between $1 \mathrm{pmol} / \mathrm{mg}$ and $10 \mathrm{pmol} / \mathrm{mg}$. Those targets that were expressed at medium level generated medium binding activity for $40.0 \%$ of the GPCRs. GPCRs with high levels of expression in Western blots, showed an equal distribution ( $44.0 \%$ each) of the specific binding in the groups for medium and high binding activity. However, some GPCRs with only weak signals in Western blots showed high functional binding activity. For example, the rat serotonin 5-HT7 receptor (5H7_RAT) generated no signal by immunodetection, but demonstrated a specific binding of $2.18 \mathrm{pmol} / \mathrm{mg}$. This might be a reflection of the ratio between functional and nonfunctional receptors. A significant effect on the binding level was seen from the expression optimization efforts conducted in $P$. pastoris. Less efforts have so far been made for optimization of SFV expression. In this context, only limited studies in three cell lines (BHK21, CHO-K1 and HEK293) were performed in parallel and also the optimal harvest time was established for each GPCR.

The two SFV vectors used in this study did not show any significant difference in expression levels for the GPCRs tested in parallel (Hassaine et al. 2006). It has also previously been demonstrated that a C-terminally fused Bio-tag had no effect on the expression levels and specific binding of the human neurokinin-1 receptor (Lundstrom et al. 1995). In contrast, some of the GPCRs expressed from the pSFV2genB and pSFV2genC vectors showed significantly lower specific binding activity than previously reported. For instance, the human $\alpha 2$-adrenergic receptor $(\alpha 2$-AR) showed specific binding of only $0.05 \mathrm{pmol} / \mathrm{mg}$ in comparison to previously published values of $176 \mathrm{pmol} / \mathrm{mg}$ (Sen et al. 2003). Likewise, the human histamine $\mathrm{H} 2$ receptor (HH2R) generated $20 \mathrm{pmol} / \mathrm{mg}$ receptor compared to another SFV-based study of $100 \mathrm{pmol} / \mathrm{mg}$ (Hoffmann et al. 2001). However, in the case of the $\alpha 2$-AR, an SFV vector with a translation enhancement signal from the SFV/capsid vector was used. This has been demonstrated to increase the expression level by $5-10$ fold (Sjöberg et al. 1994). Moreover, in the previously published study on the HH2R, ligand was added to the cell culture medium, which significantly increased the specific binding. Although these differences might explain the discrepancy in the results, also the presence of the influenza HA signal sequence as well as other tags in this study could have an effect. Interestingly, the adenosine $\mathrm{A} 2 \mathrm{~A}$ receptor generated extreme specific binding values $(287 \mathrm{pmol} / \mathrm{mg})$ from the vector with the signal sequence in comparison to $40 \mathrm{pmol} / \mathrm{mg}$ from the standard SFV vector (Lundstrom 2000).

Conclusions and future prospects

In summary, all three systems provided high expression levels of several GPCRs. The bacterial expression allowed production of large quantities of GPCRs albeit in inclusion bodies. This strategy was, however, justified by the positive development of refolding technology on GPCRs and also by the large number of targets evaluated. Expression of GPCRs in bacterial membranes requires substantial engineering of constructs and with the resources available, it was impossible to perform studies on more than 100 targets in parallel. For this reason, direct comparison between
812

813

814

815

816

817

818

819

820

821

822

823

824

825

826

827

828

829

830

831

832

833

834

835

836

837

838

839

840

841

842

843

844

845

846

847 
the success of GPCR expression in prokaryotes and eukaryotes is inappropriate. Thus, not surprisingly, the yeast- and SFV-based systems generated a much higher number of successfully expressed GPCRs than E. coli. Moreover, the functional activity could be verified by radioligand binding assays at each step of expression and purification. The post-translational modifications and particularly glycosylation was observed in both yeast and mammalian cells, but the different glycosylation patterns in the two cell types were not studied here. The effect of glycosylation on crystallization will only be discovered later, but from this aspect it might be an advantage to express the nonglycosylated GPCRs in E. coli.

The current study has provided a massive amount of information on the expression of more than 100 GPCRs in three expression systems. Although only $50 \%$ of the GPCRs were expressed in $\mathrm{i}$, all the recombinant GPCRs could be located in inclusion bodies and for some targets huge amounts, up to $350 \mathrm{mg} / \mathrm{L}$, could be produced in fermentor cultures. Plenty of material is therefore available for refolding studies and the development of improved refolding technologies. Although the purpose of this study was not to perform extensive studies on refolding, preliminary results from trials on several GPCRs suggest that receptor binding activity could be re-established, which will be communicated in future publications in detail. Similarly, several of the GPCRs expressed in $P$. pastoris were subjected to large-scale production in yeast cell cultures, which generated huge biomasses, providing material for further studies on solubilization, purification and crystallization. Likewise, well expressed GPCRs from SFV-infected mammalian suspension cultures have been subjected to solubilization and purification. Preliminary observations indicated that several GPCRs expressed both from P. pastoris vectors in yeast cells and SFV vectors in mammalian cells can be solubilized and purified to high homogeneity. Detailed results will be presented elsewhere shortly. Selected GPCRs have now been subjected to crystallization screening and optimization.

Acknowledgements We are grateful for the technical assistance provided by Juliette Kempf and Tania Steffen (UMR7175, Illkirch, France), Gabriele Maul (Max Planck Institute of Biophysics, Frankfurt, Germany), Marie-Eve Gravière and Céline Huyghe (University of Marseille, France) and Mrs. Nouzha Hassaine (BioXtal, Epalinges, Switzerland). We are also thankful for the help with E. coli scale-up and fermentation from Prof. Rainer Rudolph and Mr. Jan Oschman (Martin-Luther University, Halle, Germany). We would like to thank all those academic and industrial contributors who provided us with cDNAs for several GPCRs used in this study. The financial support received for the MePNet program from the industrial partners is acknowledged.

\section{References}

Akermoun M, Koglin M, Zvalova-Ioos D, Folschweiller N, Dowell SJ, Gearing KL (2005) Prot Expr Purific 44:65-74

André N, Cherouati N, Prual C, Steffan T, Zieder-Lutz G, Magnin T, Pattus F, Michel H, Wagner R, Reinhart C (2006) Protein Science, in press

Baneres J-L, Martin A, Hullot P, Girard JP, Rossi JC, Parello J (2003) J Mol Biol 329:801-814

Blasey H, Lundstrom K, Tate S, Bernard AR (1997) Cytotechnol 24:65-72

Cramer H, Schmenger K, Heinrich K, Horstmeyer A, Boening H, Breit A, Piiper A, Lundstrom K, Müller-Esterl W, Schroeder C (2001) Eur J Biochem 268:5449-5459

Cronan JE, Jr (1990) J Biol Chem 265:10327-10333

Guan XM, Kobilka TS, Kobilka BK (1992) J Biol Chem 267:21995-21998

Hassaine G, Wagner R, Kempf J, Cherouati N, Hassaine N, Prual C, André N, Reinhart C, Pattus F, Lundstrom K (2006) Prot Purif Expr 45:343-351

Hoffmann M, Verzijl D, Lundstrom K, Simmen U, Alewijnse AE, Timmerman H, Leurs R (2001) Eur J Pharmacol 427:105-114

Hovius R, Tairi A-P, Blasey A, Bernard A, Lundstrom K, Vogel H (1998) J Neurochem 70:824-834

Kleber-Janke T, Becker WM (2000) Protein Expr Purif 19:419424

Knight RD, Freeland SJ, Landweber LF (2001) Genome Biol 2, Epub 2001 Mar 22

Kozak M (1986) Cell 44:283-292

Lopez de Maturana R, Willshaw A, Kuntzsch A, Rudolph R, Donnelly D (2003) J Biol Chem 278:10195-10200

Lundstrom K (2000) Intervirology 43:247-257

Lundstrom K (2003) Biochim Biophys Acta 1610:90-96

Lundstrom K (2006) In: Lundstrom K, Chiu M (eds) G Proteincoupled receptors in drug discovery. CRC Press, Boca Raton USA, pp 3-14

Lundstrom K, Mills A, Buell G, Allet E, Adami N, Liljestrom P (1994) Eur J Biochem 224:917-921

Lundstrom K, Vargas A and Allet B (1995) Biochem Biophys Res Comm 208:260-266

Mazina KE, Strader CD, Fong TM (1994) J Recept Res 14:63-73

Miroux B, Walker JE (1996) J Mol Biol 260:289-298

Palczewski K, Kumasaka T, Hori T, Behnke CA, Motoshima F, Fox BA, Le Trong I, Teller DC, Okada T, Stenkamp RE, Yamamoto M, Miyano M (2000) Science 289:739-745

Schweitzer C, Kratzeisen C, Adam G, Lundstrom K, Malherbe $\mathrm{P}$, Ohresser S, Stadler H, Wichman J, Woltering T, Mutel V (2000) Neurpharmacology 39:1700-1706

Sen S, Jaakola VP, Heimo H, Engstrom M, Larjomaa P, Scheinin M, Lundstrom K, Goldman A (2003) Protein Expr Purif 32:265-275

Sjöberg M, Suomalainen M, Garoff H (1994) Bio/Technology $12: 1127-1131$

Sueoka N (1961) Cold Spring Harb Symp Quant Biol 26:35-43

Tucker J, Grisshammer R (1996) Biochem J 317:891-899

Weiss HM, Grisshammer R (2002) Eur J Biochem 269:82-92

Weiss HM, Haase W, Michel H, Reilander H (1995) FEBS Lett 377:451-456

Weiss HM, Haase W, Reilander H (1998) Methods Mol Biol 103:227-239
903

904

905

906

907

908

909

910

911

912

913

914

915

916

917

918

919

920

921

922

923

924

925

926

927

928

929

930

931

932

933

934

935

936

937

938

939

940

941

942

943

944

945

946

947

948

949

950

951

952

953

954

955

956

957

958

959

960

961

962 